\title{
Bronquio puente con agenesia del bronquio principal derecho y arteria pulmonar izquierda en hamaca: causa de estridor en niños. Reporte de un caso y revisión de la literatura
}

\author{
Martha Helena Cuellar-Santaella, $\bowtie$ María José Pecero-Hidalgo, Francisco Cuevas-Schacht, \\ Sara Alejandra Solórzano-Morales, Laura Camacho-Reyes, Eduardo Liquidano-Pérez
}

Instituto Nacional de Pediatría, Ciudad de México, México.

\begin{abstract}
RESUMEN. El bronquio puente junto con el sling de la arteria pulmonar o hamaca de la arteria pulmonar, como se conoce en nuestro medio, son anomalías congénitas ya descritas; pero poco frecuentes como causa de estridor persistente en los niños. Se describe el caso de una paciente de tres años referida por disnea, infecciones respiratorias recurrentes y probable malformación vascular. Al examinarla presentaba estridor evidente. En la broncoscopia no se observa bronquio principal derecho ni permite el paso al bronquio izquierdo y la tomografía computarizada multicorte puso en evidencia la presencia de un bronquio puente asociado a sling de la pulmonar. La presencia de ambas malformaciones se clasifica como tipo 2B según Wells y Landing; y si bien su diagnóstico sólo se realizaba post mortem en épocas pasadas, la tecnología actual permite confirmarlo de manera oportuna.
\end{abstract}

Palabras clave: Bronquio puente, arteria pulmonar izquierda en hamaca, sling de la pulmonar, hamaca pulmonar, estridor.

Bridging bronchus with agenesis of the right main bronchus and left pulmonary artery sling: cause of stridor in children. Report of a case and review of the literature

\begin{abstract}
The bridge bronchus (BP) along with the pulmonary artery sling (SAP) are already described, but infrequent, congenital anomalies as a cause of persistent stridor in children. The case of a 3-year-old patient referred to as dyspnea, recurrent respiratory infections and suspected vascular ring is described. When examining she presented stridor evident so we decided to request bronchoscopy which does not show main bronchus right nor allows to pass to the left. The multislice computed tomography demonstrates the presence of a bridge bronchus associated with pulmonary sling. The presence of both malformations is classified as type $2 \mathrm{~B}$ according to the contribution of wells and landing and although its diagnosis was mostly post-mortem in past times, the current technology allows to confirm it in a timely manner.
\end{abstract}

Key words: Bridging bronchus, pulmonary artery abnormalities, pulmonary sling, vascular rings, stridor.

\section{INTRODUCCIÓN}

La asociación entre bronquio puente (BP) y arteria pulmonar izquierda (API) en hamaca ha sido reportada en alrededor de 21 artículos en inglés y tres en español. Desde su primera descripción en $1976,{ }^{1}$ el bronquio

$\triangle$ Autor para correspondencia:

Dra. Martha Cuellar-Santaella, Instituto Nacional de Pediatría,

Ciudad de México, México.

Correo electrónico: martha.hcs12@gmail.com

Trabajo recibido: 15-VI-2018; aceptado: 18-X-2018 puente se considera como un bronquio que se origina del bronquio principal izquierdo (BPI) y se dirige hacia el lóbulo medio e inferior derecho, mientras que el bronquio principal derecho (BPD) da origen para el bronquio del lóbulo superior derecho. ${ }^{2}$ Es decir, ambos pulmones están conectados entre sí por un bronquio que se localiza en posición distal a la carina principal ${ }^{3}$ y se conoce como pseudocarina.

La API en hamaca forma parte de los anillos vasculares incompletos, en donde la arteria pulmonar izquierda se origina de la porción proximal de la rama derecha, pasa posterior a la tráquea y anterior 
al esófago para dirigirse al hilio y pulmón izquierdo, por encima del bronquio principal izquierdo. ${ }^{4}$ La presencia de estas dos malformaciones no es infrecuente y se puede asociar a compresión de la vía aérea, estenosis y traqueomalacia, ${ }^{5}$ por lo que es importante tenerlo en cuenta en pacientes con sintomatología respiratoria obstructiva a repetición, como la presencia de estridor que se documenta en esta paciente.

\section{PRESENTACIÓN DEL CASO}

Femenino a término, madre de 42 años, producto de la cuarta gestación, embarazo normoevolutivo, nace por cesárea, peso de 2,500 gramos, Apgar 8, requerimiento de oxígeno al nacer y estridor inspiratorio sin repercusión. Consultó por primera vez a los ocho meses por tos disfónica, en accesos y aumento del estridor, el cual aumentaba con la actividad física, el invierno y mejoraba al dormir, recibió nebulizaciones con adrenalina con mejoría transitoria. Continúo con cuadros de rinorrea, tos húmeda, aumento del estridor y disnea de grandes esfuerzos, en los cuales recibió broncodilatadores de acción corta, larga y esteroides sin mejoría.

Es referida a los dos años al Instituto Nacional de Pediatría (INP), Ciudad de México, por falla de medro, laringotraqueítis en varias ocasiones y ecocardiograma con defecto septal auricular tipo ostium secundum con probable ausencia de porción proximal de la rama izquierda de la arteria pulmonar. Evaluada por los servicios de Cardiología y Neu- mología de nuestro instituto, con clase funcional II, peso: $10.3 \mathrm{~kg}$, talla: $83.5 \mathrm{~cm}$, IMC: 14.9 , T/E: $89 \%$, P/T: $92 \%$, saturación al ambiente $96 \%$, frecuencia cardíaca: 98 latidos/min, frecuencia respiratoria: 24 respiraciones/min. Soplo sistólico en foco pulmonar y estridor inspiratorio.

Se realizaron estudios de imagen y endoscópico (figura 1). La broncoscopia permite observar lo que corresponde a la carina principal, en forma de quilla, nacarada a 7-8 cm de las estructuras supraglóticas y de donde emerge un esbozo que corresponde al BPD; al mismo nivel se observa el BPI de características infundibulares por donde no es posible avanzar con el videobroncoscopio BF-3C160 de $3.8 \mathrm{~mm}$ de diámetro. No hay estenosis traqueal. En retrospectiva, se observa de nuevo la radiografía de tórax PA y muestra carina principal a nivel de T4 con un pequeño bud derecho y lo que corresponde al BPI con disminución del calibre en el tercio inferior. Presencia de ambos pulmones. La ecocardiografía mostró defecto septal auricular tipo ostium secundum de $6 \mathrm{~mm}$ con emergencia de la arteria pulmonar izquierda de la arteria pulmonar derecha (figura 2).

La tomografía con reconstrucción 3D de la vía aérea confirma la alteración de la anatomía bronquial con un esbozo de BPD a la altura de T4 y termina en fondo de saco, un BPI con disminución del calibre, del cual, a nivel de T7, emerge un bronquio que se dirige hacia el hemitórax derecho dando origen a un pulmón bilobular con una cisura mayor bien formada y un esbozo de cisura menor (figura 3). Esta alteración anatómica es lo que se conoce como BP. Exactamente en el punto
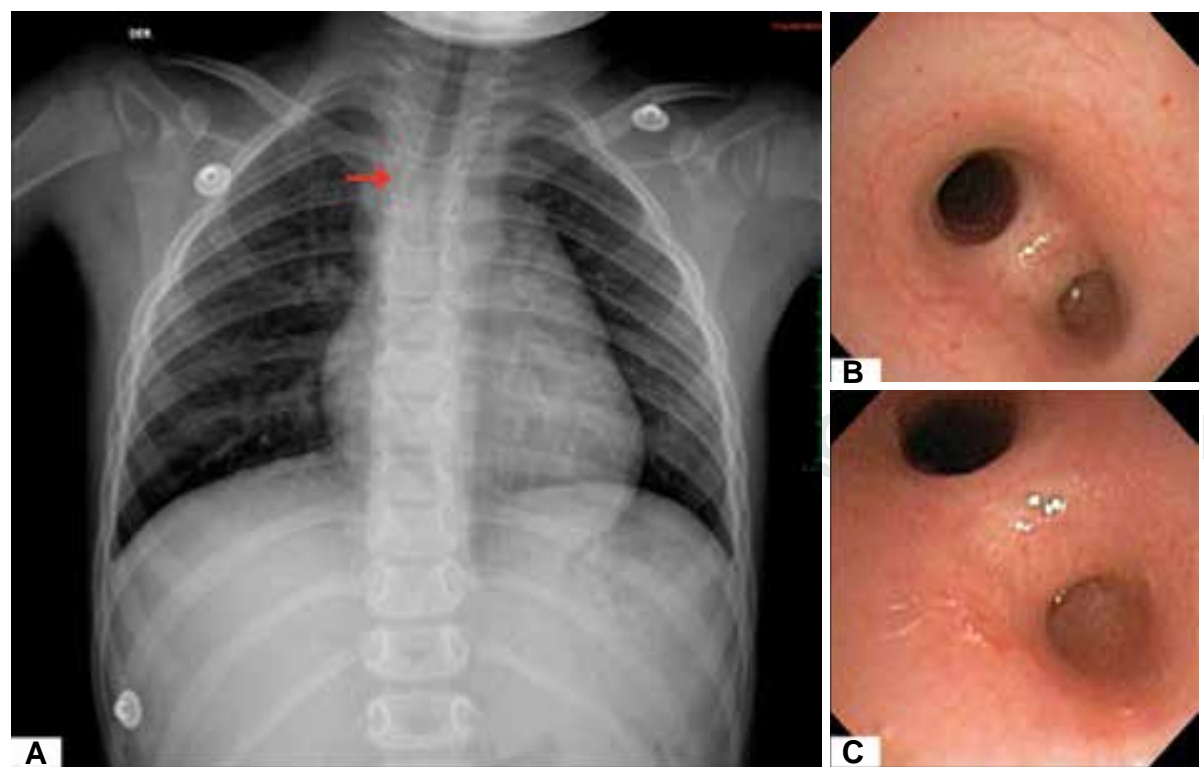

\section{Figura 1.}

A: Radiografía de tórax donde se observa disminución del calibre de la tráquea a nivel de T4. B-C: Broncoscopia con carina principal en forma de quilla, nacarada, con un bronquio principal derecho en fondo de saco y bronquio principal izquierdo, largo, de características infundibulares. 
de origen del $\mathrm{BP}$, se observa como la arteria pulmonar derecha abraza al BPI y produce una mayor disminución de su calibre.

La paciente ingresó al Servicio de Cardiología para cierre de comunicación interauricular, reinserción
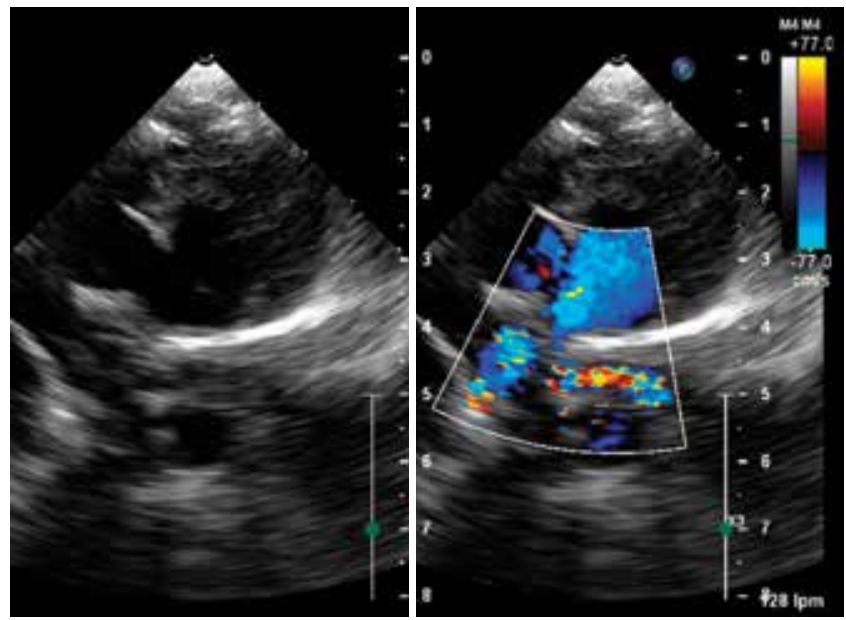

Figura 2. Imagen ecocardiográfica (corte supraesternal alto). Se observa cómo las ramas pulmonares emergen de la rama izquierda. de rama izquierda de arteria pulmonar y exploración externa de árbol bronquial. La reinserción de la API se justificó con el fin de eliminar la compresión sobre el bronquio y permitir su potencial de crecimiento. En el procedimiento no se encontró broncomalacia, desde tráquea hasta ambos bronquios (BP y BPI), pero sí con disminución en el calibre. Presentó período posoperatorio normal en la terapia cardiovascular y en hospitalización del Servicio de Cardiología. Egresó a la semana, con estridor inspiratorio leve intermitente a la auscultación, tratamiento con furosemida y espironolactona. A las seis semanas de la cirugía se realizó nueva angiotomografía donde se observa adecuada reinserción, pero permanece la disminución del calibre del BPI en la misma ubicación por donde transitaba la API en hamaca (figura 4). Actualmente, la paciente presenta estridor leve e intermitente durante grandes esfuerzos, está asintomática a nivel cardiovascular, clase funcional I y sin tratamiento médico.

\section{DISCUSIÓN}

La clasificación sistemática propuesta por Wells y Landing en 1988, permitió aclarar y unificar la rela-
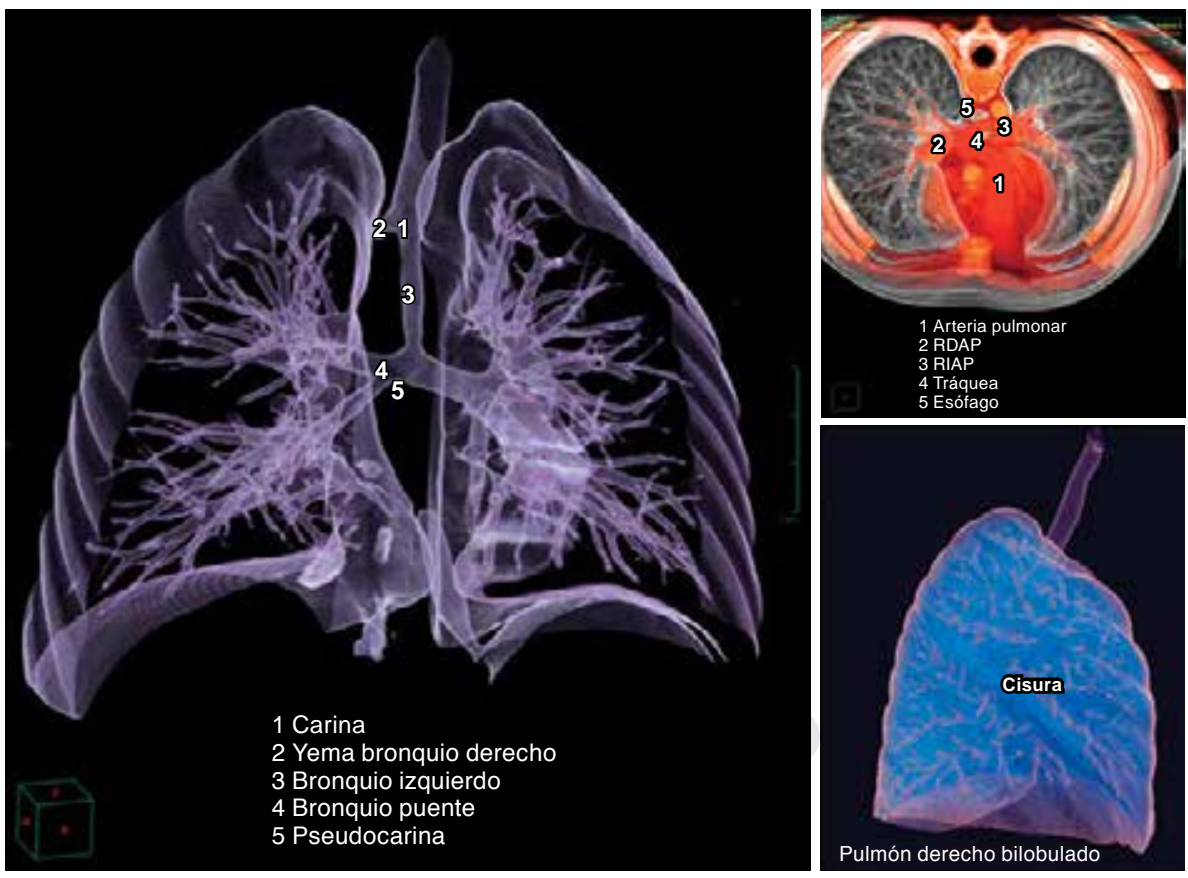

Figura 3. TC-3D: reconstrucción 3D de la vía aérea con equipo Somatom Sensation de 64 cortes evidencia carina principal a nivel de T4 con un bronquio principal derecho rudimentario y bronquio principal izquierdo en embudo, de donde emerge un bronquio puente a nivel de T7 (pseudocarina). En el corte axial se observa como la RDAP emerge de la RIAP. Observe la imagen del pulmón derecho bilobular. RDAP: rama derecha de la arteria pulmonar. RIAP: rama izquierda de la arteria pulmonar. 

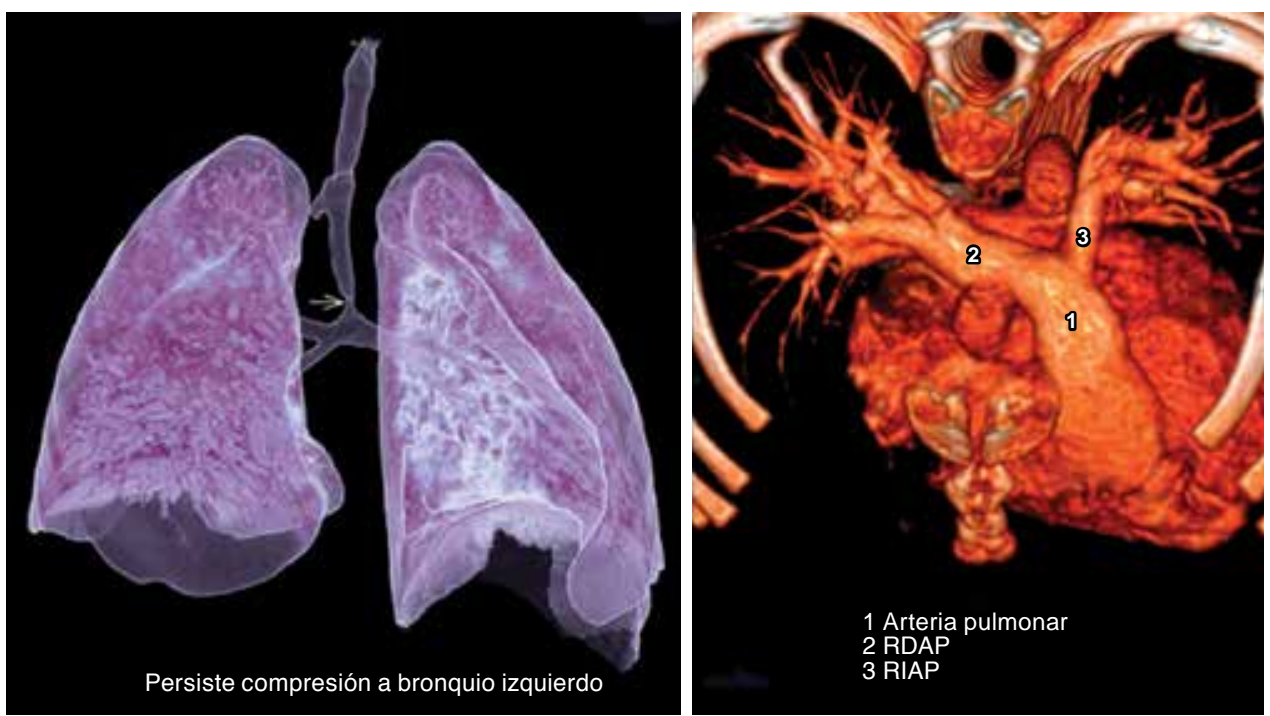

Figura 4. TC-3D: Reconstrucción 3D de la vía aérea después de la reinserción de la rama derecha de la arteria pulmonar donde aún se observa la disminución del calibre del bronquio principal izquierdo, de donde sale el bronquio puente.

1. Clasificación por Wells y Landing para arteria pulmonar en hamaca y bronquio puente

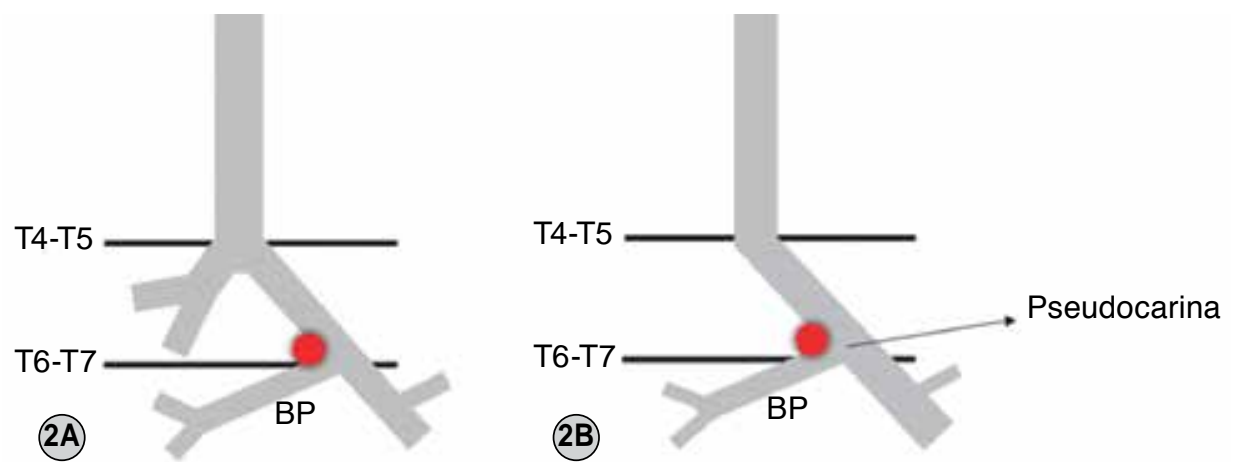

2. Tipos de bronquio puente publicados

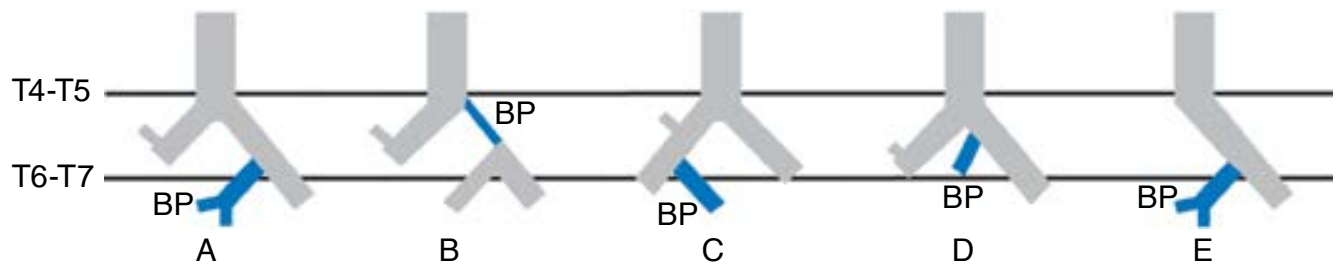

Figura 5. 1. Clasificación del bronquio puente y la arteria pulmonar en hamaca. 2. Clases de bronquio puente con o sin sling de la pulmonar.

Elaboración y diseño de figura: Fredy Arboleda Pereira.

ción entre dos malformaciones congénitas importantes: el BP y la API en hamaca o sling de la pulmonar. ${ }^{6}$ Este anillo vascular se asocia a malformaciones del árbol bronquial entre un $40-54 \%{ }^{7}$ y el BP es la malformación traqueobronquial más frecuentemente asociada (78\%), seguida de la estenosis traqueal con o sin pars membranosa. ${ }^{8}$ La clasificación explica la existencia de dos tipos de API en hamaca: las no asociadas a BP (1A y $1 \mathrm{~B})$ y las asociadas a BP $(2 \mathrm{~A}$ y $2 B)^{1}$ a las cuales nos referiremos en este artículo 
Tabla 1. Clases de BP publicados, sus asociaciones y manifestaciones.

\begin{tabular}{|c|c|c|c|c|c|}
\hline $\begin{array}{l}\text { Tipo de } \\
\text { BP }\end{array}$ & $\begin{array}{l}\text { Tipo de BP } \\
\text { con API }\end{array}$ & $\begin{array}{l}\text { Autor/año } \\
\text { Título }\end{array}$ & $\begin{array}{l}\text { Edad del } \\
\text { paciente }\end{array}$ & $\begin{array}{l}\text { Malformación } \\
\text { asociada }\end{array}$ & Cuadro clínico \\
\hline \multirow[t]{10}{*}{ A } & No aplica & $\begin{array}{l}\text { González-Crussi, } \\
1976\end{array}$ & 8 horas & - Ano imperforado & $\begin{array}{l}\text { Falla ventilatoria } \\
\text { posquirúrgica, acidosis, } \\
\text { atelectasia y estridor }\end{array}$ \\
\hline & No aplica & $\begin{array}{l}\text { Starshak R, } \\
1981\end{array}$ & 15 días & $\begin{array}{l}\text { - Ano imperforado /fístula } \\
\text { perianal-agenesia de } \\
\text { coxis/CIA /DAP } \\
\text { - Riñón en herradura } \\
\text { - } \text { Anormalidades de vertebras }\end{array}$ & $\begin{array}{l}\text { Falla ventilatoria } \\
\text { posquirúrgica }\end{array}$ \\
\hline & Paciente 1: $2 \mathrm{~A}$ & Medina G, 1992 & 2 meses & No aplica & Cianosis y apnea \\
\hline & $2 \mathrm{~A}$ & Stokes J, 1997 & 2 meses & SPA & Sibilancias y SDR \\
\hline & $\begin{array}{l}\text { Paciente 1: } 2 \mathrm{~A} \\
\text { Paciente 2: } \\
\text { No aplica } \\
\text { Paciente 3: } \\
\text { No aplica }\end{array}$ & Baden W, 2008 & $\begin{array}{l}\text { Paciente 1: } \\
\text { RN 41 sem. } \\
\text { Paciente 2: } \\
\text { RN 42 sem. } \\
\text { Paciente 3: } \\
\text { RN } 36 \text { sem. }\end{array}$ & $\begin{array}{l}\text { Paciente 1: Atresia anal, } \\
\text { displasia de sacro, FRV, } \\
\text { útero hipoplásico con } \\
\text { ausencia de ovario izquierdo, } \\
\text { TF, SAP } \\
\text { - Paciente 2: CIA/CIV/DAPVP } \\
\text { - Paciente 3: CIV y atresia } \\
\text { esofágica }\end{array}$ & No especifican \\
\hline & $2 A$ & $\begin{array}{l}\text { Du plesis, } \\
2008\end{array}$ & 5 meses & - SAP & $\begin{array}{l}\text { Obstrucción de VAI, } \\
\text { infecciones respiratorias } \\
\text { y apnea }\end{array}$ \\
\hline & No aplica & Iwataní S, 2014 & RN 40 sem. & Compresión de arteria pulmonar & Falla respiratoria \\
\hline & No aplica & $\begin{array}{l}\text { Schanabel A, } \\
2012\end{array}$ & 2 años & Ninguna & $\begin{array}{l}\text { Sibilancias, estridor, } \\
\text { disnea y tos }\end{array}$ \\
\hline & No aplica & Li D, 2017 & 10 meses & CIA /SPA & Estridor y disnea \\
\hline & $2 \mathrm{~A}$ & Carrillo, 2018 & 3 años & SAP/TF & Cianosis \\
\hline B & No aplica & Topcu S, 2006 & 10 meses & $\mathrm{CIA}$ & Sibilancias, SDR y tos \\
\hline \multirow[t]{2}{*}{ C } & No aplica & Hawass N, 1990 & Feto 18 sem. & No reportan & No reportan \\
\hline & No aplica & Wheeler D, 1998 & 6 meses & Coartación de aorta & Atelectasia crónica \\
\hline $\mathrm{D}$ & No aplica & Rishavy T, 2003 & 5 meses & Ninguna & Tos y sibilancias \\
\hline \multirow[t]{2}{*}{$E$} & $\begin{array}{l}\text { Paciente 1: 2B } \\
\text { Paciente 2: 2A } \\
\text { Paciente 3: 2B } \\
\text { Paciente 4: 2B } \\
\text { Paciente 5: 2B }\end{array}$ & Wells T, 1988 & $\begin{array}{l}\text { Paciente 1: } \\
2 \text { días } \\
\text { Paciente 2: } \\
2 \text { meses } \\
\text { Paciente 3: } \\
6 \text { meses } \\
\text { Paciente 4: } \\
11 \text { meses } \\
\text { Paciente 5: } \\
4 \text { meses }\end{array}$ & $\begin{array}{l}\text { - Paciente 1: SAP } \\
\text { - Paciente 2: SAP, ausencia } \\
\text { de vesícula, cor triatriatum. } \\
\text { Pulmón derecho monolobular } \\
\text { - Paciente 3: estenosis } \\
\text { traqueal } \\
\text { - Paciente 4: hidronefrosis, } \\
\text { FTE, membrana laríngea } \\
\text { - Paciente 5: estenosis } \\
\text { traqueal }\end{array}$ & No especifican \\
\hline & $2 B$ & Wells T, 1990 & Un día & $\begin{array}{l}\text { SAP/DSVD/-estenosis de la } \\
\text { pulmonar/CIA/CIV/AAD/EM/ } \\
\text { Cámaras cardíacas } \\
\text { izquierdas de menor } \\
\text { tamaño/pulmón derecho } \\
\text { monolobulado/ausencia de } \\
\text { vesícula }\end{array}$ & Cianosis \\
\hline
\end{tabular}


Continúa de la Tabla 1. Clases de BP publicados, sus asociaciones y manifestaciones.

\begin{tabular}{|c|c|c|c|c|c|}
\hline $\begin{array}{l}\text { Tipo de } \\
\text { BP }\end{array}$ & $\begin{array}{l}\text { Tipo de BP } \\
\text { con API }\end{array}$ & $\begin{array}{l}\text { Autor/año } \\
\text { Título }\end{array}$ & $\begin{array}{l}\text { Edad del } \\
\text { paciente }\end{array}$ & $\begin{array}{l}\text { Malformación } \\
\text { asociada }\end{array}$ & Cuadro clínico \\
\hline \multirow[t]{5}{*}{$E$} & $2 \mathrm{~B}$ & Medina G, 1992 & 6 meses & $\begin{array}{l}\text { SAP, estenosis traqueal por } \\
\text { anillos completos }\end{array}$ & Tos y disnea \\
\hline & No aplica & Gaino D, 2015 & 2 días & - Atresia pulmonar/DAP & Parámetros altos VM \\
\hline & No aplica & El-Molla A, 2016 & 10 sem. & - DSVS/TGV/CIA/CIV/VACTER & $\begin{array}{l}\text { SDR con dificultades } \\
\text { para extubación }\end{array}$ \\
\hline & $2 \mathrm{~B}$ & Wang S, 2017 & 5 meses & SAP & Tos y SDR \\
\hline & $2 \mathrm{~B}$ & Xuelei L, 2017 & Feto 21 sem. & $\begin{array}{l}\text { SAP y pulmón derecho } \\
\text { monolobulado }\end{array}$ & No aplica \\
\hline
\end{tabular}

CIA: Comunicación interauricular; DAP: Ductus arterioso persistente; SAP: Sling de la arteria pulmonar; SDR: Síndrome de dificultad respiratoria; RN: recién nacido; FRV: Fístula recto-vaginal; TF: tetralogía de Fallot; CIV: Comunicación interventricular; DAPVP: Drenaje anómalo parcial de venas pulmonares; VAI: Vía aérea inferior; FTE: Fístula traqueoesofágica; DSVD: Doble salida del VD; AAD: Arco aórtico derecho; EM: Estenosis mitral; TGV: Trasposición de grandes vasos.

(figura 2). El 2A corresponde al clásico BP donde existe una bifurcación normal en BPD y BPI en T4T5, pero el bronquio para el lóbulo medio y basal derecho provienen del BPI. El ángulo de bifurcación es normal para el BPD $\left(35^{\circ}\right)$ y reducido para el BPI $\left(25^{\circ}\right)$. El 2B es aquél donde el BPD está ausente o sólo es un divertículo, por lo que el árbol bronquial del pulmón derecho proviene en su totalidad del BPI. Tanto en el $2 \mathrm{~A}$ y $2 \mathrm{~B}$, el origen del BP se encuentra a nivel de T6-T7 y es en este punto donde se ubica la API en hamaca. ${ }^{1}$ Los ángulos de bifurcación del BP están aumentados para el brazo derecho (entre $65^{\circ}$ y $76^{\circ}$ ) y normales para el izquierdo. Es precisamente esta alteración lo que se interpreta como una $T$ invertida o pseudocarina. ${ }^{8}$ Para el momento en que se propuso la clasificación, el tipo 2B era el más común con 19 pacientes $(51 \% \text { de los reportados) })^{1}$ y desde 1988 sólo hay cuatro reportes para esta clasificación $n^{9,7,10,11}$ y seis casos para el tipo $2 \mathrm{~A} .{ }^{7,8,12-15} \mathrm{Si}$ bien se sabe que los anillos vasculares representan sólo el $1 \%$ de los defectos congénitos, por la frecuencia de asociación con BP, es de esperarse un mayor número de reportes de esta malformación bronquial, pero no todos los pacientes presentan síntomas que sugieran la presencia de compresión extrínseca de la vía aérea, por lo que no se les realizan estudios endoscópicos o de imagen.

De lo publicado sobre BP, existen cinco clases y el presente caso sería tipo $\mathrm{E}$, el cual es el segundo en frecuencia (figura 5, tabla 1). En el INP, es el cuarto caso de BP asociado a API en hamaca desde su primer reporte en 1992, uno tipo $2 \mathrm{~A}$ y otro $2 \mathrm{~B}$, ambos con diagnóstico post mortem $;{ }^{7}$ durante el 2018 fueron dos casos incluyendo el presente, uno $2 \mathrm{~A}$ y éste $2 \mathrm{~B}$.
A diferencia de los demás pacientes descritos en la literatura, nuestra paciente no presentaba alteraciones costovertebrales ni gastrointestinales y los últimos dos casos han sido con diagnóstico en vida, muy diferente a la concepción inicial de este padecimiento, donde se llegó a considerar incompatible con la vida; ${ }^{3}$ sin embargo, en la actualidad, se ofrecen técnicas de reparación quirúrgicas exitosas. ${ }^{16}$ La mortalidad depende del grado de obstrucción de la vía aérea, de las malformaciones mayores que le acompañen y de diagnóstico oportuno. Aunque la relación está bien establecida, sigue siendo un hallazgo raro, por lo que vale la pena documentarlo.

De los síntomas respiratorios que pueden presentarse en esta asociación, el estridor es el más común como signo de obstrucción de la vía aérea y estuvo presente como síntoma principal en nuestra paciente. ${ }^{17}$ Se define como un sonido áspero y vibratorio de paso variable de aire causado por la obstrucción parcial de las vías respiratorias que produce un flujo turbulento a través de éstas. ${ }^{17}$ Aunque el estridor puede ser el resultado de un proceso relativamente benigno, también puede ser el primer signo de un trastorno grave e incluso mortal y exige atención inmediata y una evaluación exhaustiva para descubrir la etiología. ${ }^{18}$ Existen varias causas conocidas de estridor, la presentación aguda está más relacionada con procesos inflamatorios o infecciosos. ${ }^{19}$ En el caso de nuestra paciente fue de forma crónica y se relaciona con alteraciones anatómicas o patología funcional. ${ }^{19}$

Se cree que las anomalías cardiovasculares congénitas se asocian significativamente con las anomalías congénitas y adquiridas de las vías respiratorias. Al- 
gunos estudios indican que casi todos los niños con cardiopatía o anomalías vasculares, que presentan un síntoma de vía aérea, tendrán un resultado positivo importante en la endoscopia. ${ }^{20}$

Es importante recalcar que todo niño con estridor debe estudiarse, el objetivo de la historia clínica y el examen físico es evaluar la gravedad del estridor y decidir si se requiere intervención inmediata para asegurar la vía aérea o continuar con la evaluación endoscópica y radiológica. ${ }^{3}$ En la búsqueda de la etiología, la broncoscopia y la tomografía con reconstrucción tridimensional son complementarias. La broncoscopia permite corroborar la permeabilidad de la vía aérea y establecer el diagnóstico de certeza sobre la bifurcación del árbol bronquial. Si no se realiza, las imágenes radiológicas del remanente del BPD pueden ser confundidas con el remanente de un bronquio traqueal y se interpretaría que la verdadera carina se encuentra a un nivel inferior (T6) de lo habitual, cuando en realidad corresponde al nacimiento del BP o lo que han Ilamado como pseudocarina.

\section{CONCLUSIONES}

El estudio de los niños con estridor siempre debe incluir la visualización endoscópica de la vía aérea y ante la visualización de malformaciones en el árbol bronquial, se debe descartar anomalías en los grandes vasos y el corazón para otorgar diagnósticos y tratamientos oportunos. Para este caso en particular, el estridor se podía explicar por la compresión de la arteria pulmonar en hamaca sobre el origen del BP (pseudocarina); pero al realizar la reinserción del anillo vascular, la paciente aún presentaba estridor leve, el cual puede explicarse por la disminución fija del diámetro del BPI como quedó demostrado en la angiotomografía de control. Se hará seguimiento y plan expectante ante los síntomas de la paciente.

\section{Conflicto de intereses}

Los autores declaran no tener conflicto de intereses.

\section{REFERENCIAS}

1. Wells TR, Gwinn JL, Landing BH, Stanley P. Reconsideration of the anatomy of sling left pulmonary artery: the association of one form with bridging bronchus and imperforate anus. Anatomic and diagnostic aspects. J Pediatr Surg 1988;23(10):892-898.

2. Vargas BMC, Varón $\mathrm{H}$, Pérez $A M C$, Puerta RAF, Rúales FFL. Anormalidades congénitas y adquiridas de la tráquea y bronquios principales en la población pediátrica. Rev Colomb Radiol 2011;22(1):3095-3103.

3. Pérez LF. Malformaciones congénitas broncopulmonares. Toma de decisiones para diagnóstico, clasificaciòn y tratamiento. En: Pérez LF, Cuevas F, editores. Neumología y cirugía de tórax pediátrica. INP: Ciudad de México; 2013.p.467-494.

4. Andrés S, Moreno IC. Anillos vasculares y slings. En: Albert DC, editor. Cardiología pediátrica y cardiopatías congénitas del niño y del adolescente. 2da ed. Madrid: Grupo CTO; 2010.p. 473-482. Citado: 21 septiembre, 2017. Accesible en: http://www.secardioped.org/ readcontents.php?file=webstructure/lp_cap33. pdf\&op=download

5. Palavecino BT, Palma CR. Bronquio cardíaco accesorio y otras variantes de la anatomía tráqueo-bronquial. Rev Chil Enferm Respir 2017;33(2):131-136.

6. Berdon WE, Muensterer OJ, Zong YM, Backer CL. The triad of bridging bronchus malformation associated with left pulmonary artery sling and narrowing of the airway: the legacy of Wells and Landing. Pediatr Radiol 2012;42(2):215-219. doi: 10.1007/s00247-011-2273-2.

7. Medina-Escobedo G, Lopez-Corella E. Sling left pulmonary artery, bridging bronchus, and associated anomalies. Am J Med Genet 1992;44(3):303-306.

8. Baden W, Schaefer J, Kumpf M, et al. Comparison of imaging techniques in the diagnosis of bridging bronchus. Eur Respir J 2008;31(5):1125-1131. doi: 10.1183/09031936.00045907.

9. Wells TR, Stanley P, Padua EM, Landing BH, Warburton D. Serial section-reconstruction of anomalous tracheobronchial branching patterns from CT scan images: bridging bronchus associated with sling left pulmonary artery. Pediatr Radiol 1990;20(6):444-446.

10. Wang SY, Gao W, Zhong YM, et al. Multislice computed tomography assessment of tracheobronchial patterns in partial anomalous left pulmonary artery. J Comput Assist Tomogr 2017;41(6):983-989. doi: 10.1097/ RCT.0000000000000623.

11. Li X, Li X, Saul D, Zhao Z. Fetal MRI diagnosis of 2 types of left pulmonary artery sling. Radiol Case Rep 2017;12(4):653-657. doi: 10.1016/j.radcr.2017.07.013.

12. Li D, Feng $Y$, Hirata $Y$, An Q. Sling pulmonary artery with bridging bronchus and narrowing airway: A case report. Ann Thorac Cardiovasc Surg 2017;23(5):265-267. doi: 10.5761/atcs.cr.17-00006.

13. Inoue N, Oka N, Miyaji K. Univentricular heart with bridging bronchus and sling left pulmonary artery. Pediatr Cardiol 2013;34(5):1280-1282. doi: 10.1007/ s00246-012-0387-4.

14. Huang SC, Wu ET, Wang CC, et al. Repair of complex tracheobronchial stenosis with left pulmonary artery sling and bridging bronchus. Ann Thorac Surg 2010;90(4):1379-1381. doi: 10.1016/j. athoracsur.2009.11.070.

15. du Plessis AM, Andronikou S, Goussaard P. Bridging bronchus and sling left pulmonary artery: a rare entity demonstrated by coronal CT with 3-D rendering display 
and minimal-intensity projections. Pediatr Radiol 2008;38(9):1024-1026. doi: 10.1007/s00247-008-0913-y.

16. Ragalie WS, Chun RH, Martin T, et al. Side-toside tracheobronchoplasty to reconstruct complex congenital tracheobronchial stenosis. Ann Thorac Surg 2017;104(2):666-673. doi: 10.1016/j. athoracsur.2017.01.011.

17. Zoumalan R, Maddalozzo J, Holinger LD. Etiology of stridor in infants. Ann Otol Rhinol Laryngol 2007;116(5):329-334.
18. Spear L, Deane SA. Differential diagnosis of stridor in children. Emerg Med 2009;41(9):10-19.

19. Parente-Hernández A, García-Casillas MA, Matute JA, et al. ¿Es el estridor un síntoma banal en el lactante? An Pediatr 2007;66(6):549-644. doi: 10.1157/13107389.

20. Guillemaud JP, El-Hakim H, Richards S, Chauhan N. Airway pathologic abnormalities in symptomatic children with congenital cardiac and vascular disease. Arch Otolaryngol Head Neck Surg 2007;133(7):672676. 